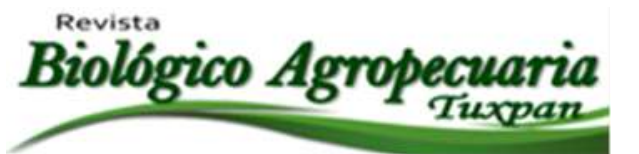

\title{
Exploración de beneficios de vainilla en el Área Natural Protegida Sierra de Otontepec, Veracruz en busca de coliformes
}

\author{
Exploration of vanilla benefits in the Sierra Otontepec Protected Natural Area, Veracruz in search of \\ coliforms
Ramírez Juárez Sharon ${ }^{1}$, Elorza Martínez Pabloํㅜ, García Muñoz Silvia Amanda², Villareal Ramírez Víctor H. ${ }^{2}$, Uranga Valencia Luisa P. ${ }^{2}$

${ }^{1}$ Facultad de Ciencias Biológico Agropecuarias, Universidad Veracruzana. ${ }^{2}$ Facultad de Ciencias Agrotecnológicas, Universidad Autónoma de Chihuahua.

๑utor para correspondencia: pelorza@uv.mx

Recibido: $15 / 04 / 2019$
Aceptado: 15/05/2019

\section{RESUMEN}

La vainilla mexicana (Vanilla planifolia Andrews) es utilizada en la industria agroalimentaria, refresquera, licorera, farmacéutica, cosmética, tabacalera y artesanal por su excelente calidad aromática con notas dulces e intensas y olor a tabaco con un carácter cremoso (Tenailleu et al., 2004). Su beneficiado es un proceso de conservación del fruto, que se realiza mediante la deshidratación y fermentación del fruto verde sin aroma al fruto beneficiado, el cual adquiere un olor suave y agradable ante nuestro paladar. Al analizar las muestras de los cinco sitios ubicados en la Sierra de Otontepec se pudo comprobar que no se obtuvo presencia de colonias precursoras de coliformes dando negativa esta prueba, este resultado fue comparado con lo que marca la NOM-182-SCFI-2011 dentro de los parámetros de especificaciones microbiológicas para la vainilla, lo que refleja que no hay resto de microorganismos dañinos en vainas, obteniendo vainas de muy buena calidad. Todas las muestras obtenidas de los cinco sitios tienen las mismas condiciones, en cuanto a manejo y procesamiento de las vainas en el beneficio además de tener las mismas características de suelo y agua.

Palabras clave: Calidad, Vanilla planifolia Andrews, Especificaciones.

\begin{abstract}
Mexican vanilla (Vanilla planifolia Andrews) is used in the food industry, soft drink, liquor, pharmaceutical, cosmetics, tobacco and craft for its excellent aromatic quality with sweet and intense notes and smell of tobacco with a creamy character (Tenailleu et al., 2004). Its benefit is a process of conservation of the fruit, which is done by dehydrating and fermenting the green fruit without aroma to the beneficiated fruit, which acquires a soft and pleasant smell before our palate. When analyzing the samples from the five sites located in the Sierra de Otontepec it was possible to verify that no presence
\end{abstract}


of coliform precursor colonies was found, giving negative this test, this result was compared with what the NOM-182-SCFI-2011 marks within the parameters of microbiological specifications for vanilla, which shows that there are no remaining harmful microorganisms in pods, obtaining pods of very good quality. All the samples obtained from the five sites have the same conditions, in terms of handling and processing the pods in the benefit as well as having the same soil and water characteristics.

Keywords: Quality, Vanilla planifolia Andrews, Specifications.

\section{INTRODUCCIÓN}

La vainilla mexicana (Vanilla planifolia Andrews) es utilizada en la industria agroalimentaria, refresquera, licorera, farmacéutica, cosmética, tabacalera y artesanal por su excelente calidad aromática con notas dulces e intensas y olor a tabaco con un carácter cremoso (Tenailleu et al., 2004).

El beneficiado de vainilla es un proceso de conservación del fruto, que se realiza mediante la deshidratación y fermentación del fruto verde sin aroma al fruto beneficiado, el cual adquiere un olor suave y agradable ante nuestro paladar. Los totonacas practicaban el beneficiado y ha sido mejorado a través del tiempo por productores mexicanos, italianos y franceses (Reyes et al., 2008).

Los microorganismos coliformes constituyen un grupo heterogéneo con hábitat primordialmente intestinal para la mayoría de las especies que involucra. Cuando los coliformes llegan a los alimentos, no sólo sobreviven, sino que se multiplican, por lo que en los alimentos el grupo coliforme adquiere un significado distinto al que recibe en el agua. En productos alimenticios que han recibido un tratamiento térmico (pasteurización, horneado, cocción, etc.), se utilizan como indicadores de malas prácticas sanitarias (Camacho et al., 2009).

La NOM-113-SSA1-1994 explica que los microorganismos coliformes son el grupo que más se utiliza en la microbiología de alimentos como indicador de prácticas higiénicas inadecuadas.

El uso de los coliformes como indicador sanitario es aplicado para:

- La detección de prácticas sanitarias deficientes en el manejo y en la fabricación de los alimentos. - La evaluación de la calidad microbiológica de un producto, aunque su presencia no necesariamente implica un riesgo sanitario.

- Evaluación de la eficiencia de prácticas sanitarias e higiénicas del equipo.

- La calidad sanitaria del agua y hielo utilizados en las diferentes áreas del procesamiento de alimentos.

- La demostración y la cuenta de microorganismos coliformes, puede realizarse mediante el empleo de medios de cultivos líquidos o sólidos con características selectivas o diferenciales.

La vainilla puede presentar contaminación por agentes bacterianos, virales y especies fúngicas, causando pérdidas significativas en los frutos verdes. Algunos de los géneros aislados más importantes son Azotobacter sp., Enterobacter sp., Fusarium, Rhizoctonia y Colletotrichum (Odoux, 2011).

\section{MATERIALES Y MÉTODOS}

El área natural protegida de la Sierra Otontepec, se ubica dentro de la región huasteca, al norte del estado de Veracruz $\left(21^{\circ} 11^{\prime}\right.$ y $21^{\circ} 40^{\prime}$ 
de latitud norte y $97^{\circ} 52^{\prime}$ y $98^{\circ} 05$ de longitud oeste), a una altura de 30 a $1300 \mathrm{msnm}$. Representa una formación montañosa aislada de la Sierra Madre Oriental (SEFIPLAN, 2014).

En esta área se realizó el cultivo y el beneficiado de vainilla por el método de congelación, para posteriormente analizar la presencia de microorganismos coliformes.
Las muestras de vainas fueron recolectadas en cinco sitios pertenecientes al área natural protegida de la Sierra Otontepec, Veracruz. De las cuales cuatro sitios realizaron el beneficiado por congelamiento en vainas durante seis meses, mientras que un sitio realizo el beneficiado en un año y seis meses, contando con diferentes tipos de tutor, como se muestra a continuación:

Cuadro 1. Tutores de vainilla.

\begin{tabular}{|c|c|c|}
\hline SITIO & DURACION DE CONGELAMIENTO & TUTOR \\
\hline Estación & Un año y medio & Espalderas + malla sombra \\
\hline Tamalinillo & Seis meses & Citrus aurantifolia \\
\hline Xochitlan & Seis meses & Erythrina \\
\hline Tancoco & Seis meses & Bursera simaruba+citrus \\
\hline Agua salada & Seis meses & Citrus sinensis \\
\hline
\end{tabular}

Las vainas se mantuvieron dentro de una cámara de congelación a $-4{ }^{\circ} \mathrm{C}$, después de cumplir el periodo determinado para cada uno de los sitios, se descongelaron, posteriormente se realizaron 35 soles; es decir sudado y secado al sol para obtener el beneficiado y después evaluar así en laboratorio el efecto que causa la duración del congelamiento entre los contenidos de vainillina y microorganismos coliformes.

Para realizar la determinación de coliformes se esterilizó material de cristalería (matraz, filtro y pinzas) durante 2 horas en un horno en seco, esterilizando también el área de trabajo. Para obtener la muestra a analizar, en 1 litro de agua destilada y con ayuda de una micro pipeta se retiró 1 mililitro de esa solución, reemplazándolo por 1 mililitro de muestra homogenizándose perfectamente.
En la parte superior del aparato por el que pasa un filtro se agregó con pinzas pequeñas un Millipore sterilized, sobreponiéndole un contenedor de vidrio donde se unió con una pinza para mantenerlo fijo, una vez listo se encendió la bomba de vacío para eliminar cualquier presencia de aire y poco a poco fue vaciándose la muestra obtenida. El millipore sterilized es una membrana cuadriculada que absorbe la solución al pasar por el filtro que cae en el matraz.

Una vez filtrado el litro de muestra, se apagó la bomba de vacío y se retiró la cristalería que se unió con las pinzas, con ayuda de unas pinzas pequeñas se retiró el millipore, depositándolo en una caja Petri y agregándole una sustancia la cual agilizó el tiempo para observar en dado caso la producción de colonias. 
Este procedimiento se realizó con las 4 muestras siguientes, se dejaron en una cámara de incubación a una temperatura de entre $34-36^{\circ} \mathrm{C}$ durante 12 horas.

Transcurridas las 12 horas se revisaron las cajas para ver si existía algún desarrollo de colonias.

\section{RESULTADOS Y DISCUSIÓN}

Realizando la determinación de las muestras obtenidas de los cinco sitios, no se obtuvo presencia de colonias precursoras de coliformes dando negativa esta prueba, este resultado fue comparado con lo que marca la NOM-182SCFI-2011 dentro de los parámetros de especificaciones microbiológicas para la vainilla, lo que refleja que no hay resto de microorganismos dañinos en vainas, obteniendo vainas de muy buena calidad. Todas las muestras obtenidas de los cinco sitios tienen las mismas condiciones, en cuanto a manejo y procesamiento de las vainas en el beneficio además de tener las mismas características de suelo y agua.

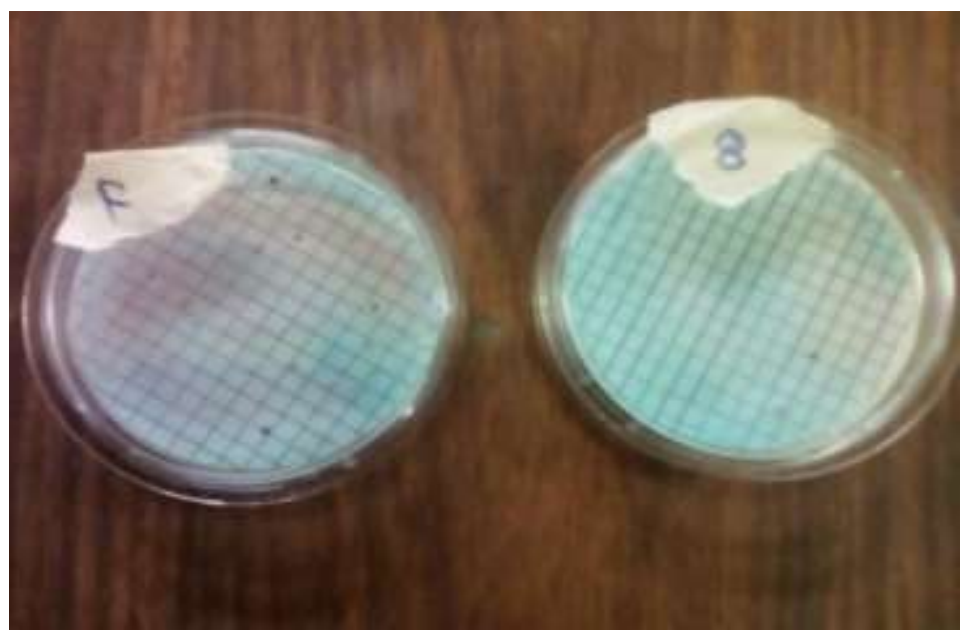

Figura 1. Cajas Petri sin presencia de colonias coliformes

\section{CONCLUSIÓN}

Al realizar la determinación de microorganismos coliformes no se mostró presencia de colonias precursoras de coliformes en las muestras, lo que indica un aspecto importante en la calidad que tiene la vainilla que se cultiva y beneficia en la sierra Otontepec, Veracruz.

\section{LITERATURA CITADA}

Camacho, A; Giles, M; Ortegón, A; Palao, M;

Serrano, B; Velázquez, O. 2009. Técnicas para el análisis microbiológico de alimentos. $2^{\circ}$ edición. Facultad de química, UNAM. México.

Odoux, E. 2011. Vanilla Curing. En: Odoux E, Grisoni M (eds). Vanilla. (Medicinal and aromatic plants-industrial profiles). CRC Press. Boca Raton Florida. 173-185. https://doi.org/10.1201/EBK1420083378

Reyes, L.; Rodríguez M.B; Kelso, B. H; Huerta, L. M; Ibáñez, M.A. 2008. Beneficiado tradicional de vainilla. México. Benemérita Universidad Autónoma de Puebla. Puebla, México. 
SEFIPLAN, Secretaría de Finanzas y Planeación Social del Estado de Veracruz (2014). Cuadernillos municipales.

Tenailleu E.J; Lancelin P; Robins R.J; Akoka S.
2004. Authentication of the origin of vanilling using Quantitative Natural Abundance C NMR. Journal of Agricultural and Food Chemistry. 52: 7782- 7787.

https://doi.org/10.1021/jf048847s

Copyright (c) 2019 Sharon Ramirez Juárez, Pablo Elorza Martinez, Silvia Am anda Garcia Muñoz, Victor H. Villarreal Ramirez y Luisa P. Uranga Valencia

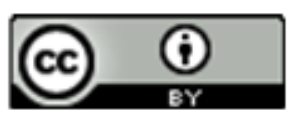

Este texto está protegido por una licencia licencia CreativeCommons 4.0.

Usted es libre para Compartir — copiar y redistribuir el m aterial en cualquier medio o formato-y Adaptar el documento - remezclar, transformar y crear a partir del material- para cualquier propósito, incluso para fines com erciales, siempre que cumpla la condición de:

Atribución: Usted debe dar crédito a la obra original de manera adecuada, proporcionar un enlace a la licencia, e indicar si se han realizado cambios. Puede hacerlo en cualquier forma razonable, pero no de forma tal que sugiera que tiene el apoyo del licenciante o lo recibe por el uso que hace de la obra.

Resumendelicencia - Textocompletodelalicencia 